www.jmscr.igmpublication.org

Impact Factor 5.84

Index Copernicus Value: 83.27

ISSN (e)-2347-176x ISSN (p) 2455-0450

crossref DOI: _https://dx.doi.org/10.18535/jmscr/v5i8.104

Journal Of Medical Science And Clinical Research

Original Article

\title{
Diagnostic role of ultrasonography for diagnosis of acute abdomen
}

\author{
Authors \\ Dr Vivekanand Rai ${ }^{1}$, Dr Mayank Mishra ${ }^{2}$, Dr Yash Pandey ${ }^{3}$, Dr Puspendra Singh ${ }^{4}$ \\ Dr Alok Tripathi ${ }^{5}$ \\ ${ }^{1}$ Associate Professor, ${ }^{2,5}$ Assistant Professor, ${ }^{3,4}$ Senior Resident \\ HIMS Varanasi INDIA
}

\begin{abstract}
Introduction: The primary aim of this study is to evaluate the diagnostic accuracy, sensitivity and specificity of sonography in the evaluation of acute abdomen, given that it involves no ionizing radiation and excels in the depiction of acute gynaecologic conditions in young women, and during pregnancy. Clinical diagnosis is based primarily on symptoms and physical findings, but is often difficult to establish; up to 50\% of patients hospitalized for possible appendicitis do not actually have this disorder Authors of large prospective studies report a 22-30\% removal rate of normal appendices at surgery. Ultrasonography has established itself as an invaluable tool in surgery. In surgical practice, abdominal pain is perhaps the most common symptom encountered and almost in every case of abdominal pain surgeon prefers to use the ultrasound to confirm the diagnosis. Although physical examination of the patient is the most important part in proper diagnosis many a time some positive help is required in the form of investigations especially ultrasonography.

Methods: This was a study of hundred patients carried out at a tertiary care hospital admitted with complaints of severe abdominal pain except those with a history of trauma or with a history of chronic abdominal pain. Clinical history, physical examination, ultrasonography, per operative findings and histopathological examination were used to come to a final conclusion.

Result: In this study ultrasonography was diagnostic in $78 \%$ of patients. Two patients were misdiagnosed and in 20 patients other investigations were required for the confirmation of diagnosis. The sensitivity and specificity of ultrasound in diagnosing acute appendicitis, renal calculus, liver abscess, mesenteric lymphadenitis, acute pancreatitis and ovarian cyst was $100 \%$ and in calculus cholecystitis it was $93.75 \%$ and $100 \%$ respectively.

Conclusion: Ultrasonography is superior in organ system imaging. It helps in showing organ specific lesions and its accurate measurement which is helpful in follow up and response to treatment. Ultrasonography is also helpful in diagnosing alternative disease and to reduce negative laparotomy rate.

Keywords: Abdominal Pain, Acute Abdomen, Diagnosis,.
\end{abstract}

\section{Introduction}

The primary aim of this study is to evaluate the diagnostic accuracy, sensitivity and specificity of sonography in the evaluation of acute abdomen, given that it involves no ionizing radiation and excels in the depiction of acute gynaecologic conditions in young women, and during pregnancy. Clinical diagnosis is based primarily 
on symptoms and physical findings, but is often difficult to establish; up to $50 \%$ of patients hospitalized for possible appendicitis do not actually have this disorder Authors of large prospective studies report a $22-30 \%$ removal rate of normal appendices at surgery.

Of the several outstanding technological advances in all branches of medicine, perhaps the most outstanding is ultrasonography. Ultrasound has established itself as an invaluable tool in surgery. Its application is over expanding due to excellent work executed in several specialized institutes and clinics.

Ultrasound should be added to the testing protocol for acute appendicitis, since it is an inexpensive, painless examination with high sensitivity and specificity. The depiction of the acute abdomen with sonographic signs of inflammation is a powerful diagnostic element in patients.

Abdomen is rightly known as "magic box". The term "acute abdomen" refers to signs and symptoms of abdominal pain and tenderness, a clinical presentation that often requires emergency surgical therapy. This challenging clinical scenario requires a thorough and expeditious workup to determine the need for operative intervention and initiate appropriate therapy. It goes without saying how important it is to make the diagnosis as early as possible in these conditions.

In surgical practice, abdominal pain is perhaps the most common symptom encountered, and almost in every case of abdominal pain the surgeon prefers to go for ultrasonography to confirm the diagnosis. Only one quarter of patients who have previously been classified with an acute abdomen actually receive surgical treatment, so the clinical dilemma is if the patients need surgical treatment or not and, furthermore, in which cases the surgical option needs to be urgently adopted. ${ }^{[1]}$ Although physical examination of the patient is the most important part in proper diagnosis, many a time some positive help is required in the form of investigations. For this added help, ultrasonography plays a great role. ${ }^{[2]}$
Ultrasonography is cheap, non-invasive, reliable, simple to perform, has no contraindications and can be repeated as and when required. It is a highresolution imaging technique. Its versatility and real-time imaging capability are also major advantages. Another unique advantage is the Doppler ultrasound, which allows visualization of blood flow and assessment of flow dynamics. Ultrasound units are now smaller and more portable, so they are widely used in multiple medical settings, including the bedside, operative suite, emergency room, and in diagnostic and interventional radiology suits. Miniaturized highresolution transducers also facilitate laparoscopic and endoscopic procedures.

\section{Materials and Methods}

This was a prospective study of 100 patients between the age of 10-70years (52 males and 48 females) carried out at Heritage institute of medical sciences invaranasi during a period of 1 year.

Inclusion Criteria: Only those non traumatic patients who were admitted in the ward with complaints of severe abdominal pain.

Exclusion Criteria: Patients with a history of trauma and with chronic abdominal pain.

The equipment was real-time ultrasonographic apparatus with a video graphic scanner (Toshiba) which uses a frequency of $3.75 \mathrm{MHz}$ for abdominal ultrasonography and $5 \mathrm{MHz}$ for transvaginal and trans-rectal ultrasonography.

All the 100 admitted patients were examined in the ward and provisional clinical diagnosis was made by the information obtained from clinical history and physical examination. Simultaneously, routine laboratory and radiological investigations were carried out.

In this study, all the patients were examined by radiologists with the pre-requisite of nil per oral from last night and bowel preparation was done and also the co-relation of clinical history with physical findings and ultrasonographic findings, ultrasonographic diagnosis was done. 
Out of the 100 patients, 60 patients were managed conservatively while the rest 40 patients were operated. Operative findings were noted and fluid or tissue collected pre or per operatively were sent for histo-pathological examination and the report was noted down Final diagnosis was made after the surgery and histo-pathological report.

\section{Results}

Sensitivity and Specificity of Ultrasonography in Diagnosis of Disease

\begin{tabular}{|l|c|c|c|c|}
\hline & $\begin{array}{c}\text { No. of } \\
\text { Cases }\end{array}$ & $\begin{array}{c}\text { No. of cases } \\
\text { where USG was } \\
\text { helpful }\end{array}$ & Sensitivity & Specificity \\
\hline Appendicitis & 22 & 22 & $100 \%$ & $100 \%$ \\
\hline $\begin{array}{l}\text { Calculus } \\
\text { Cholecystitis }\end{array}$ & 14 & 13 & $93.75 \%$ & $100 \%$ \\
\hline Renal Calculus & 17 & 17 & $100 \%$ & $100 \%$ \\
\hline Liver Abscess & 11 & 11 & $100 \%$ & $100 \%$ \\
\hline $\begin{array}{l}\text { Mesenteric } \\
\text { Lymphadenitis }\end{array}$ & 7 & 7 & $100 \%$ & $100 \%$ \\
\hline $\begin{array}{l}\text { Acute } \\
\text { Pancreatitis }\end{array}$ & 4 & 4 & $100 \%$ & $100 \%$ \\
\hline Ovarian Cyst & 3 & 3 & $100 \%$ & $100 \%$ \\
\hline Miscellaneous & 13 & 12 & $92.30 \%$ & $98.85 \%$ \\
\hline
\end{tabular}

According to the above results, ultrasonography is highly sensitive and specific for diagnosis of prevalent pathologies of acute abdominal conditions and almost gold standard.

Overall Diagnostic Accuracy of Ultrasonography in Acute Abdominal Conditions

\begin{tabular}{|l|c|c|}
\hline USG & No. of patients & Percentage \\
\hline Diagnostic & 78 & $78 \%$ \\
\hline Mis-diagnostic & 2 & $2 \%$ \\
\hline Other investigations required & 20 & $20 \%$ \\
\hline
\end{tabular}

In this study ultrasonography was diagnostic in $78 \%$ of patients. Two patients were misdiagnosed and in 20 patients other investigations were required for confirmation of diagnosis.

\section{Discussion}

In this study, the ultrasonographic diagnosis proved to be correct in $100 \%$ cases of liver abscess. However in one case of portal hypertension ultrasonography misled us and gave the differential diagnosis of splenic mass/abscess/ cyst which proved to be wrong on further study. Ultrasonography is highly accurate in gall bladder conditions, except in one case of CBD stone where the diagnosis of thrombus tumor in portal vein was made which proved to be wrong. The sensitivity and specificity of ultrasonography in diagnosing pancreatic conditions is $100 \%$. In cases of gastritis, no specific pathology was found on ultrasonography. In mesenteric lymphadenitis, ultrasonography accurately diagnosed the condition and all patients were managed conservatively. In appendicitis, it gave an accurate diagnosis in all the cases.

There are a few studies which have looked at the various parameters we analyzed. Al Ajerami ${ }^{[3]}$ in his study on acute appendicitis found the overall sensitivity and specificity of ultrasound, using surgical outcome as the gold standard, to be $84.8 \%$ and $83.3 \%$ respectively. Allemann et al ${ }^{[4]}$ reported that in USG done by surgeons for patients with acute abdominal pain the correct diagnostic rate from 348 patients $(70 \%)$ to 414 patients $(83 \%)$. In the same study, USG was found to have a sensitivity and specificity of $94 \%$ and 99\% in diagnosing biliary tract disease. Mishra et $\mathrm{al}^{[5]}$ in their study of imaging for acute abdomen had 13 cases of appendicitis. USG was diagnostic in 11 with sensitivity and specificity of $91.6 \%$ and $97 \%$. Zoller et $\mathrm{al}^{[6]}$ in their meta analysis demonstrated that USG has sensitivity of $85 \%$ and a specificity of $96 \%$ in diagnosing acute appendicitis. McGrath et $\mathrm{al}^{[7]}$ in their study on the role of early USG in the management of the acute abdomen concluded that it is most useful in the diagnosis of gynecological disorders. Manfredi et al ${ }^{[8]}$ concluded that USG in acute pancreatitis is a good screening test in patients with suspected biliary pancreatitis and a mild clinical course but contrast enhanced CT is preferred for patients with acute pancreatitis.

A prospective study was carried out by Caterino et al ${ }^{[9]}$ covering 301 patients during 4 years in the Ist Institute of Surgery at the University of Rome. After immediate clinical evaluation, an ultrasonographic examination was performed in each patient. After follow up, patients were divided into following groups: 
- Group A: Diagnosis was made by sonographic examination which had not been clinically expected.

- Group B: Ultrasonography confirmed the first diagnosis clinically suspected.

- Group C: Ultrasonography revealed the 2nd or 3rd differential diagnosis to be correct.

- Group D: Ultrasonography made no contribution to the diagnosis.

The comparison of the two studies is as follows:

\begin{tabular}{|l|c|c|}
\hline Group & Our study (100 patients) & $\begin{array}{c}\text { Caterino's study (301 } \\
\text { patients) }\end{array}$ \\
\hline Group-A & 20 patients (20\%) & 38 patients (12.7\%) \\
\hline Group-B & 60 patients (60\%) & 161 patients (53.3\%) \\
\hline Group-C & 18 patients (18\%) & 23 patients (7.7\%) \\
\hline Group-D & 2 patients (2\%) & 77 patients (25.5\%) \\
\hline
\end{tabular}

Findings of the 2 studies are almost similar in group A, B and C. However there is disparity in group $\mathrm{D}$. The difference is mostly due to variation in the total number of patients studied in both the studies. The result of this study demonstrates the usefulness of emergency ultrasonography in acute abdominal conditions involving various organ systems and associated pathologies.

Results obtained show that ultrasonography is highly accurate. In majority of the systems, a definite diagnosis was made.

\section{Conclusion}

Ultrasonography is cheap, non-invasive, reliable, simple to perform, has no contraindications and can be repeated as and when required. It also allows complete portability so that studies can easily be carried out at the bed side, in the emergency room in case of critically ill patients and even in the operating room.

For the abdominal surgeon, ultrasound provides a vital diagnostic and management aid in the assessment of the intra-abdominal diseases. It has a very high accuracy in cases of acute abdomen. Ultrasonography is superior in organ system imaging. It helps in showing organ specific lesions and its accurate measurement which is helpful in follow up and response to treatment. Ultrasonography is also helpful in diagnosing alternative disease and to reduce negative laparotomy rate.

\section{Funding: Nil}

Conflict of interest: None initiated.

Permission from IRB: Yes

\section{References}

1. Cartwright SL, Knudson MP: Evaluation of acute abdominal pain in adults. Am Fam Physician. 2008 Apr 1;77(7):971-8. [PubMed]

2. Stoker J, van Randen A, Laméris W, Boermeester MA. Imaging patients with acute abdominal pain. Radiology. 2009 Oct;253(1):31-46. doi: 10.1148/radiol.2531090302. [PubMed]

3. Al-Ajerami Y. Sensitivity and specificity of ultrasound in the diagnosis of acute appendicitis. East Mediterr Health J. 2012 Jan;18(1):66-9. [PubMed]

4. Allemann $\mathrm{F}$, Cassina $\mathrm{P}$, Rothlin $\mathrm{M}$, Largiader F: Ultrasound scans done by surgeons for patients with acute abdominal pain. Eur J Surg 1999 Oct;165(10):966-70. [PubMed]

5. Mishra DS, Magu S, Sharma N, Rattan $\mathrm{KN}$, Tiwari AD, Rohilla S. Imaging in acute abdomen. Indian J Pediatr. 2003 Jan;70(1):15-9. [PubMed]

6. Zoller WG, Kellner H, Schwerk WB. Value of ultrasound in diagnosis of acute appendicitis. Bildgebung 1996 Jun;63(2):78-82. [PubMed]

7. McGrath FP, Keeling F. The role of early sonography in the management of the acute abdomen ClinRadiol. 1991 Sep;44(3):172-4. [PubMed]

8. Manfredi R, Brizi MG, Canade A, Vecchioli A, Marano P. Imaging of acute pancreatitis. Rays 2001 Apr-Jun; 26(2):135-42. [PubMed] 
9. Caterino S, Meli C, Capotondi C, Cavallini M, Zerilli M, Schiffino L, Murante G, Alessi G, Cavallaro A. Role of ultrasonography in emergency surgery. Ann ItalChir. 1995 Jan-Feb;66(1):87-97. [PubMed] 